\title{
High-power-density capillary discharge plasma columns for shorter wavelength discharge-pumped soft-x-ray lasers
}

\author{
J. J. Gonzalez, M. Frati, and J. J. Rocca* \\ Department of Electrical and Computer Engineering, Colorado State University, Fort Collins, Colorado 80523 \\ V. N. Shlyaptsev \\ Department of Applied Sciences, UC Davis-Livermore, Livermore, California 94550 \\ and Lawrence Livermore National Laboratory, Livermore, California 94550
}

A. L. Osterheld

Lawrence Livermore National Laboratory, Livermore, California 94550

(Received 4 June 2001; published 24 January 2002)

\begin{abstract}
We report the generation of plasma columns in gas-filled capillary channels using discharge excitation powers that exceed those of previous studies by one to two orders of magnitude. Current pulses up to $200 \mathrm{kA}$ and $10-90 \%$ rise time of $\sim 10 \mathrm{~ns}$ (current increase rate $\sim 1.5 \times 10^{13} \mathrm{~A} / \mathrm{s}$ ) were utilized to excite plasmas in 3.3 and $4 \mathrm{~mm}$ diameter channels. Time resolved soft-x-ray spectra and pinhole images of the plasma were obtained. The experimental data and its comparison with model computations suggest that dense argon plasma columns $300 \mu \mathrm{m}$ in diameter with electron temperatures $>250 \mathrm{eV}$ have been obtained. These characteristics make these plasmas of interest for extending discharge-pumped lasers to shorter wavelengths.

DOI: 10.1103/PhysRevE.65.026404

PACS number(s): 52.80.- s, 42.55.-f
\end{abstract}

\section{INTRODUCTION}

There is significant interest in the development of practical soft-x-ray lasers for applications [1]. Excitation approaches include the use of high power lasers and fast capillary discharges [1-10]. Fast capillary discharges have been demonstrated to efficiently generate highly uniform plasma columns of small diameter $[11,13]$, in which large soft-x-ray amplification has been obtained by collisional excitation of Ne-like ions at wavelengths ranging from 46.9 to $60.8 \mathrm{~nm}$ $[2,6,9]$. In the particular case of Ar discharges the gain-length product for the $3 p{ }^{1} S_{0}-3 s{ }^{1} P_{1}$ transition of Ne-like $\mathrm{Ar}$ at $46.9 \mathrm{~nm}$ has exceeded saturation [7], allowing for the generation of laser output pulse energies approaching $1 \mathrm{~mJ}$ and average powers up to $3.5 \mathrm{~mW}$ [10]. Large amplification was also demonstrated in Ne-like $\mathrm{Cl}$ at $52.9 \mathrm{~nm}$ [9] and in Nelike $\mathrm{S}$ at $60.8 \mathrm{~nm}$ [6]. These lasers required the generation of plasma columns with electron densities of the order of 5 $\times 10^{18} \mathrm{~cm}^{-3}$ and electron temperatures in the range of $60-80$ eV $[2,6,7]$. Such conditions were achieved utilizing fast current pulses with peak amplitude between 23 and $40 \mathrm{kA}$ $[2,6,7,9]$. There is interest in the possible extension of this laser pumping scheme to shorter wavelengths. However, this requires the generation of significantly hotter and denser plasma columns $[12,13]$, which in turn demands a substantial scaling of the excitation power density.

Herein we report the generation and study of capillary discharge plasma columns created utilizing discharge powers between one and two orders of magnitude larger than those used in previous experiments with gas-filled capillaries. The plasma columns were generated utilizing current pulses with $10-90 \%$ rise time of about $10 \mathrm{~ns}$ and peak amplitude of up

\footnotetext{
*Email address: rocca@engr.colostate.edu
}

to $200 \mathrm{kA}$. Utilizing this discharge, Ar plasma columns were generated in capillaries with diameters of $3.3 \mathrm{~mm}$ and $4 \mathrm{~mm}$ made of either alumina $\left(\mathrm{Al}_{2} \mathrm{O}_{3}\right)$ or polyacethal $\left(\mathrm{CH}_{2} \mathrm{O}\right)_{n}$ filled with $1.3 \mathrm{~T}$ of Ar gas. As discussed below, the discharges in ceramic capillaries produced plasmas with a slightly higher degree of ionization. Since plasma columns with maximum degree of ionization are of most interest for the development of shorter wavelength discharge-pumped lasers, the majority of the data presented in this paper corresponds to ceramic capillaries. The plasmas were diagnosed using time resolved soft-x-ray spectroscopy and pinhole camera images. The dynamic of the plasma was also studied with numerical simulations. The plasma columns obtained are significantly hotter and denser than those reported in all previous gas-filled capillary experiments $[2-6,12,13]$. The next sections of the paper discuss the generation of the highpower-density capillary discharge plasma columns and the plasma diagnostic measurements, followed by an interpretation of the results based on model simulations.

\section{EXPERIMENTS}

Fast current pulses were generated through the capillary load utilizing a new type of pulsed power generator composed of three pulse compression stages. The first two stages consist of a conventional Marx generator and coaxial water capacitor that has the purpose of rapidly charging the third and final pulse compression stage. The eight-stage Marx generator is capable of operating at voltages up to $800 \mathrm{kV}$. The second compression stage, which consists of a $26 \mathrm{nF}$ coaxial water capacitor, is charged in about $1 \mu \mathrm{s}$. In turn this water capacitor is discharged through a self-breakdown spark gap pressurized with $\mathrm{SF}_{6}$ gas to charge the third and final stage in about 75 ns. This rapid charging avoids breakdown of the water-dielectric, and minimizes the charge leakage to ground 
(a)
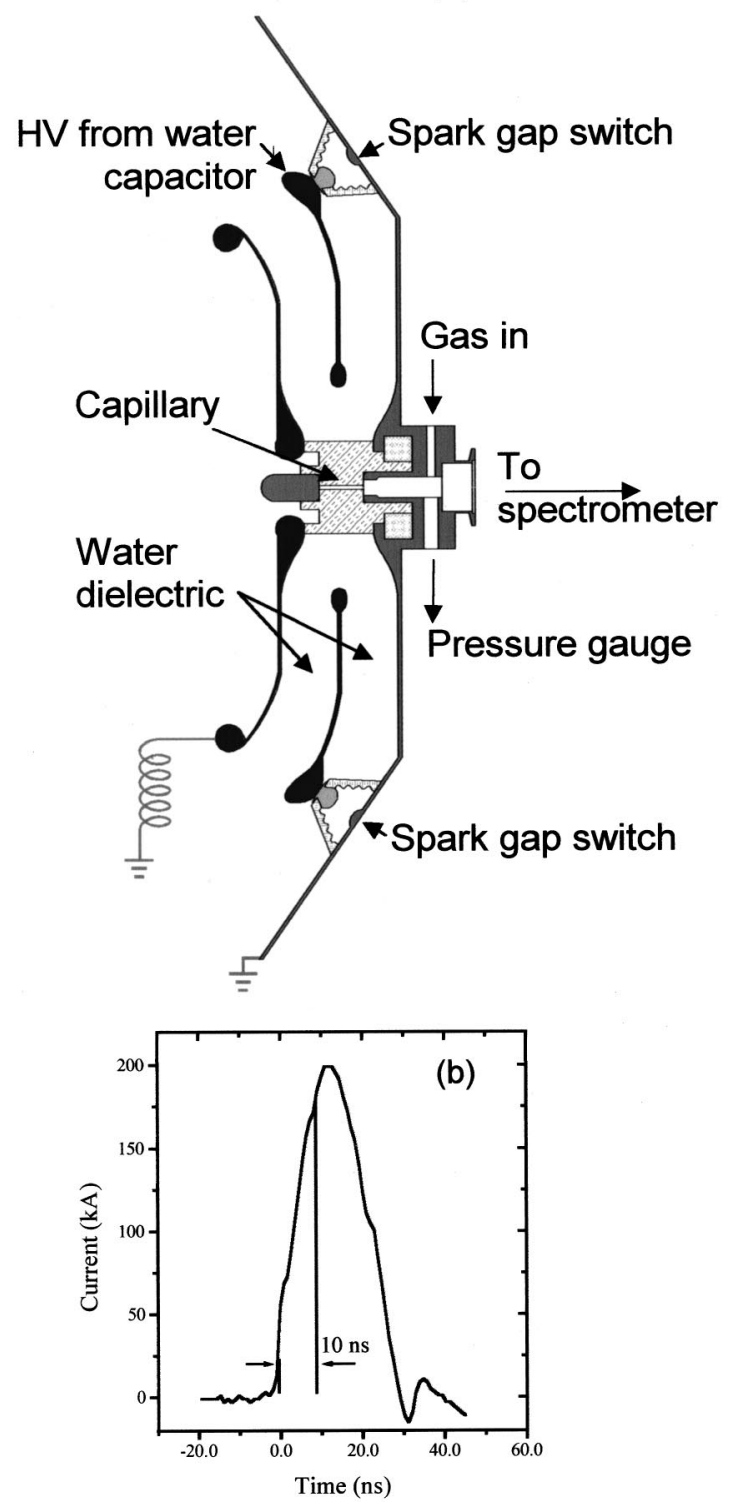

FIG. 1. (a) Schematic representation of the third pulse compression stage, consisting of a radial Blumlein transmission line switched by an array of seven synchronized spark-gap switches. (b) Current pulse.

through the finite resistant of the water used as dielectric in the transmission line of the final compression stages. The third stage, illustrated in Fig. 1(a), is of different design and consists of two radial water dielectric transmission lines connected in a Blumlein configuration. The fast current pulse that excites the capillary plasma is produced by discharging the Blumlein transmission line through an array of seven synchronized triggered spark-gap switches distributed along the outer diameter of the water transmission line. This circular array of gas pressurized spark gaps approximates a large single multichannel spark gap, allowing for a very rapid switching of the Blumlein. The capillary load is placed in the axis of the Blumlein, which together with the spark gap array defines a very low inductance loop that allows for the generation of very fast current rise times, exceeding 1.5 $\times 10^{13} \mathrm{~A} / \mathrm{s}$. The ground electrode is designed to have a central hole that allows for the observation of the axially emitted plasma radiation. Figure 1(b) illustrates a current pulse having a peak current of $200 \mathrm{kA}$ and a $10-90 \%$ rise time of $\sim 10 \mathrm{~ns}$. The current pulse was measured with a Rogowsky coil having a response risetime of less than $1 \mathrm{~ns}$. These pulse characteristics significantly exceed those of previous experiments with gas-filled capillary discharges $[2,12,13]$. Immediately preceding the fast discharge pulse the capillary channels were preionized with a current pulse of 20-40 A and 10 $\mu$ s duration. Typically several tens of shots were made with each capillary. The gas was injected utilizing a pulsed valve and was evacuated through a differential pumping system that made use of two turbomolecular pumps.

The radial evolution of the plasma columns was studied by means of an on-axis pinhole camera. A $90 \mu \mathrm{m}$ pinhole was utilized to image the plasma over the detection plane with a magnification of $3.5 \times$. The gated detection system, consisting of a microchannel plate (MCP) intensified charge coupled device (CCD) array detector, had a time resolution of $\sim 4 \mathrm{~ns}$. Wavelength discrimination was achieved utilizing one of two different sets of filters in front of the pinhole camera. Figure 2 shows a sequence of time resolved pinhole images of the soft-x-ray emitting region of the plasma for wavelengths mainly below $3 \mathrm{~nm}$. The images correspond to a $4 \mathrm{~mm}$ diameter ceramic capillary excited by current pulses of about $190 \mathrm{kA}$ peak current. Wavelength discrimination was obtained using a stack composed of a $1 \mu \mathrm{m}$ thick carbon filter and a $0.2 \mu \mathrm{m}$ thick aluminum filter. The carbon filter alone transmits photons below $3 \mathrm{~nm}$, and also in a window between about 4.5 and $10 \mathrm{~nm}$. The addition of the $\mathrm{Al}$ filter decreases the transmissivity in this window by a factor that increases from about $6 \times$ at $4.5 \mathrm{~nm}$ to about $600 \times$ at $10 \mathrm{~nm}$ as compared with the carbon filter alone, significantly enhancing the relative contribution to the images of photons with wavelengths below $3 \mathrm{~nm}$. The sequence of images shows the formation of a strong shock that originates near the capillary walls and evolves to form a compressed plasma column at the center of the capillary. The earliest image, obtained at $20 \mathrm{~ns}$ from the beginning of the current pulse, shows a cylindrical shell with an outer diameter of $\sim 800 \mu \mathrm{m}$ caused by the current distribution determined by electromagnetic field diffusion from the wall toward the axis. The subsequent images show the rapid continuous compression of the plasma column, which reaches a minimum diameter of about $300 \mu \mathrm{m}$ at $\sim 25 \mathrm{~ns}$ after the beginning of the current pulse. Subsequently the plasma column expands, and the intensity of the radiation emitted within this spectral range decreases rapidly. At times after $31 \mathrm{~ns}$ the intensity drops below the detection limit. It should be noticed that the asymmetry observed in the wings of the profiles corresponding to the larger diameter images is caused by an imperfect alignment of the pinhole rather than by an asymmetry in the plasma column. Figure 3 shows a series of pinhole images for discharge conditions similar to those of Fig. 2, but obtained utilizing only the $1 \mu \mathrm{m}$ thick carbon filter. The removal of the aluminum filter changes the range of detected wavelengths significantly enhancing the contribution from 

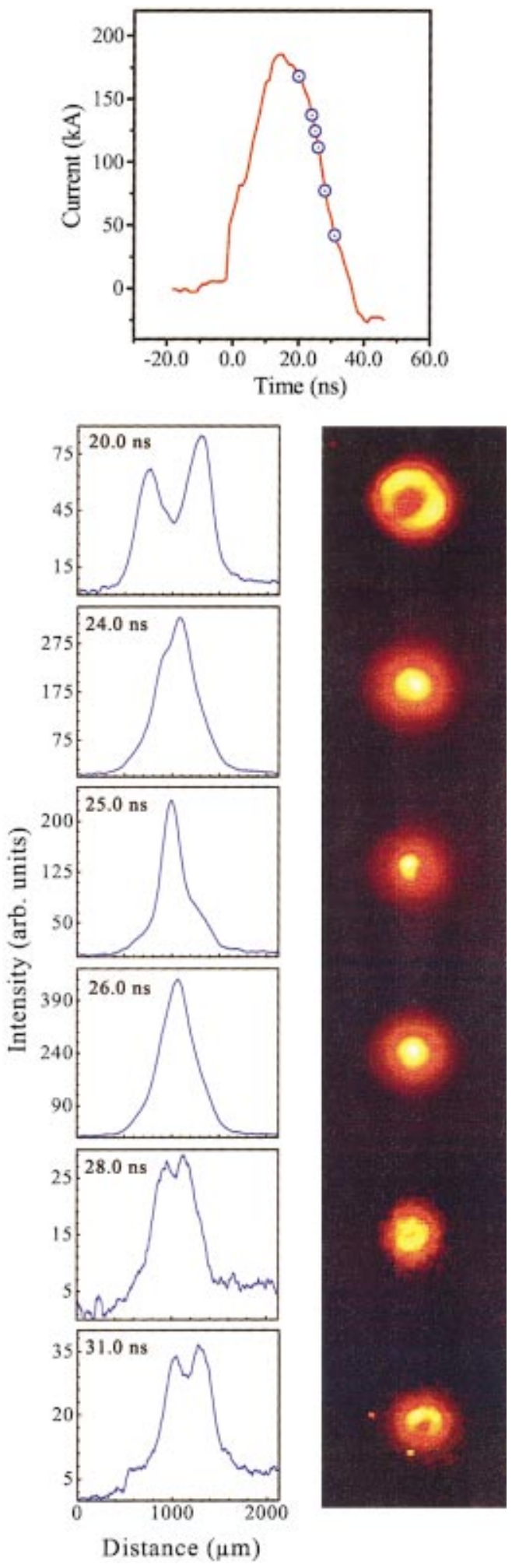

FIG. 2. (Color) Sequence of time resolved pinhole camera images obtained with discharge current pulses of $\sim 190 \mathrm{kA}$ with a $10-90 \%$ rise time of $11 \mathrm{~ns}$ through a $4 \mathrm{~mm}$ diameter ceramic capillary. The images were taken using a $1 \mu \mathrm{m}$ thick $\mathrm{C}$ filter plus a 0.2 $\mu \mathrm{m}$ thick Al filter. The image corresponds mainly to photons with wavelength below $3 \mathrm{~nm}$.
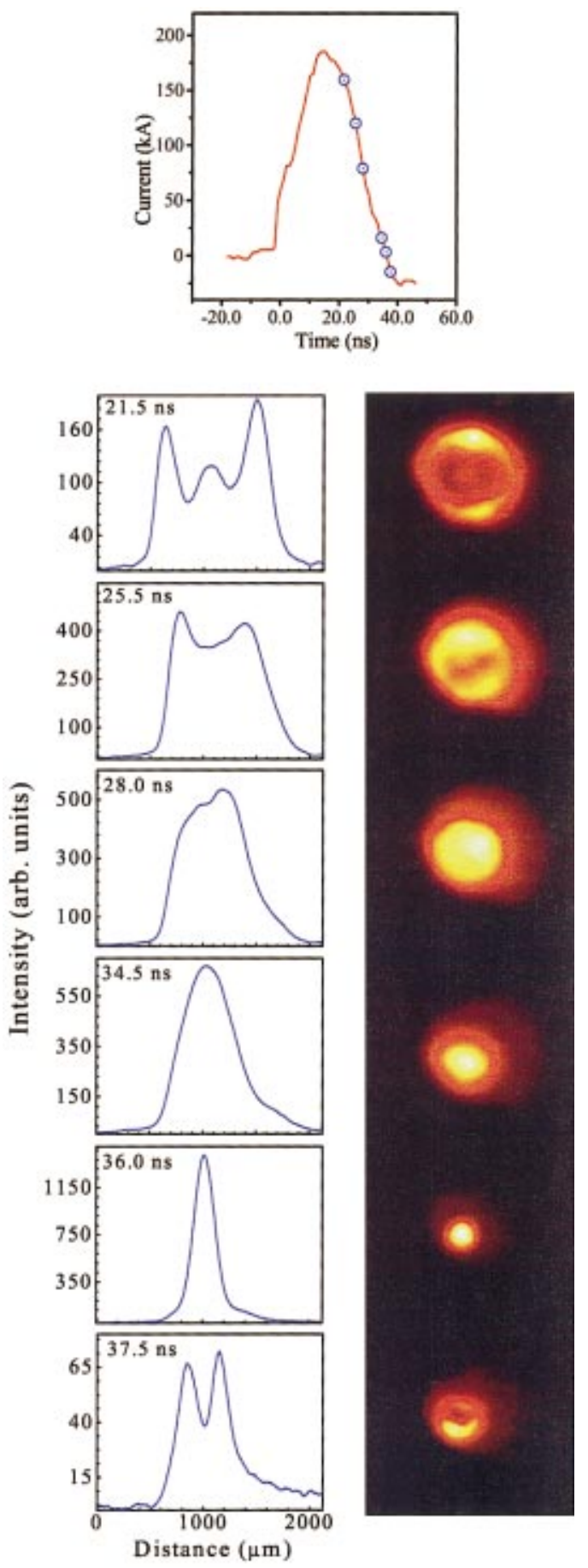

FIG. 3. (Color) Sequence of time resolved pinhole camera images of the plasma for the same discharge conditions of Fig. 2 taken using only a $1 \mu \mathrm{m}$ thick $\mathrm{C}$ filter. The filter transmits photons below $3 \mathrm{~nm}$ and also in the range between 4.5 and $10 \mathrm{~nm}$.

photons in the 4.5-10 $\mathrm{nm}$ spectral region. In this case the pinch at about $25 \mathrm{~ns}$ is largely concealed by the overlap with stronger emission at longer wavelengths, that at this time occurs in a much larger plasma region. However, Fig. 3 clearly shows the occurrence of a latter pinch that takes place around $36 \mathrm{~ns}$ from the beginning of the current pulse. This weaker second pinch was not observed with the stack of the two filters and therefore corresponds to a colder plasma. Fig- 


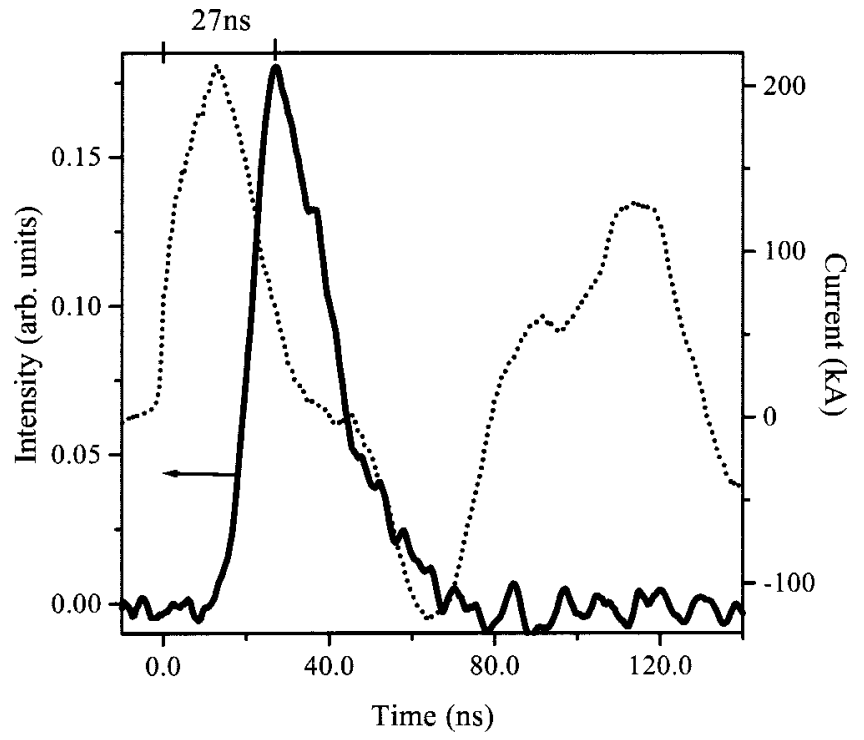

FIG. 4. Correspondence between the evolution current pulse and soft-x-ray emission measured with an MCP intensified vacuum photodiode. The data was obtained using a $1 \mu \mathrm{m}$ thick $\mathrm{C}$ filter plus a $0.2 \mu \mathrm{m}$ thick Al filter. The image corresponds mainly to photons with wavelength below $3 \mathrm{~nm}$.

ure 4 shows a different measurement of the time evolution of the radiation obtained with an MCP intensified vacuum photodiode and a filter stack composed of a $1 \mu \mathrm{m}$ thick $\mathrm{C}$ filter and a $0.2 \mu \mathrm{m}$ thick Al filter. This measurement shows that in the $4 \mathrm{~mm}$ diameter capillaries the maximum emission occurs $\sim 27$ ns after the initiation of the current pulse.

Time resolved spectroscopy was carried out through selected intervals in the spectral range between 3 and $30 \mathrm{~nm}$ to study the evolution of the degree of ionization of the plasma. The radiation axially emitted by the plasma was analyzed using a $1 \mathrm{~m}$ grazing incidence spectrograph having a 1200 $1 / \mathrm{mm}$ gold coated grating placed at $85.8^{\circ}$. A cylindrical mirror placed at a grazing incidence angle of $2^{\circ}$ was used to collect the radiation and focus it into the entrance slit of the spectrograph. The time resolved spectra were recorded utilizing a gated MCP/CCD detector with a temporal resolution of $\sim 5 \mathrm{~ns}$. The spectral range between 18 and $23 \mathrm{~nm}$, which contains spectral lines belonging to all the ionization stages between Ar XI through Ar XV, was found to be the most suitable to study the time evolution of the plasma. Figures 5(a) and 5(b) show typical spectra corresponding to a time near the occurrence of maximum ionization for polyacethal and ceramic capillaries, respectively. They correspond to plasma columns generated using current pulses with $197 \mathrm{kA}$ peak amplitude and a 10-90\% rise time of $11 \mathrm{~ns}$. The dominance in the ceramic capillaries of the Ar XV line at $22.11 \mathrm{~nm}$ (identified with an arrow in the spectra), and the larger intensity ratio between adjacent $18.795 \mathrm{~nm} \mathrm{ArXIV}$ and $18.882 \mathrm{~nm}$ Ar XI lines visible at the left of the spectra in Fig. 5(b), are indicative of a larger degree of ionization.

The series of time resolved spectra in Figs. 6 and 7 provides information of the temporal variation of the degree of ionization of the plasma for discharges in $4 \mathrm{~mm}$ and $3.3 \mathrm{~mm}$ diameter ceramic capillaries, respectively. The peak ampli- tude and rise time of the current pulses were maintained approximately constant for these two series at $190 \pm 5 \mathrm{kA}$ and $11.5 \pm 1.5 \mathrm{~ns}(10-90 \%)$, respectively. The variation of the intensity of the Ar XV line relative to the Ar XIV lines and the changing ratio of intensities between the two adjacent lines of Ar XIV and Ar XI at the left of the spectra give again an indication of the variation of the degree of ionization. This line intensity ratio peaks around the time corresponding to the first pinch, identified in the pinhole images in the vicinity of $25 \mathrm{~ns}$. Figure 7 shows that the Ar XV line is almost absent at $18.4 \mathrm{~ns}$ after the beginning current pulse and begins to dominate approximately at $21.6 \mathrm{~ns}$ after the initiation of the current pulse.

The high intensity of the ArXV line in the ceramic capillaries suggests that lines corresponding to Ar XVI ions might also be present. Line emission was indeed observed at several wavelengths corresponding to Ar XVI lines in the 3-30 $\mathrm{nm}$ range. However, the presence of these lines could not be confirmed due to their overlap with first or second order lines from Ar ions of lesser charge. Nevertheless, the theoretical calculations discussed below predict the existence of Ar XVI ions under the operating conditions of this capillary discharge. These simulations, performed with the code RADEX [10-13], show that in the case of steady-state ionization equilibrium calculated for the average value of the electron density over the time of collapse, the Ar XV ions reach maximum abundance at a temperature of $\sim 160-180 \mathrm{eV}$. In such ionization equilibrium conditions, slightly larger temperatures $T_{e} \sim 180-200 \mathrm{eV}$ are required for the relative intensities of Ar XIV and Ar XV lines to be similar to those observed in the experiment. However, the ionization equilibrium values usually represent only an estimate of the lower limit of the real temperature. In the dynamic situation of the experiment, in which the limited hot plasma lifetime is shorter than the ionization time for the high- $Z$ species present in the plasma, the temperature necessary for the creation of such spectra is notably larger. Transient ionization and hydrodynamic calculations done with RADEX, indicate the maximum electron temperature required to generate such spectra must be larger than $250 \mathrm{eV}$. This suggests the possibility of using fast high current capillary discharges for generating hightemperature plasmas approaching the $\mathrm{keV}$ range.

\section{MODEL SIMULATIONS}

The evolution of the plasma column was modeled with the one-dimensional radiative hydrodynamic-atomic code RADEX [13-15]. The model includes two temperature hydrodynamics equations, Maxwell's equations, and multicomponent atomic kinetics and radiation transport. The physical model remains in most respects similar to the one which was applied to the study of capillary discharge soft-x-ray lasers $[7,12-14]$ and microcapillary plasmas $[15]$ in recent years, but includes the atomic kinetics of the highly ionized species needed to reproduce the experimentally observable characteristics of this much higher power capillary discharge. It also contains a description of wall ablation for either polyacethal or ceramics capillaries, which includes the use of the equations of state for the solid material and the plasma, and 

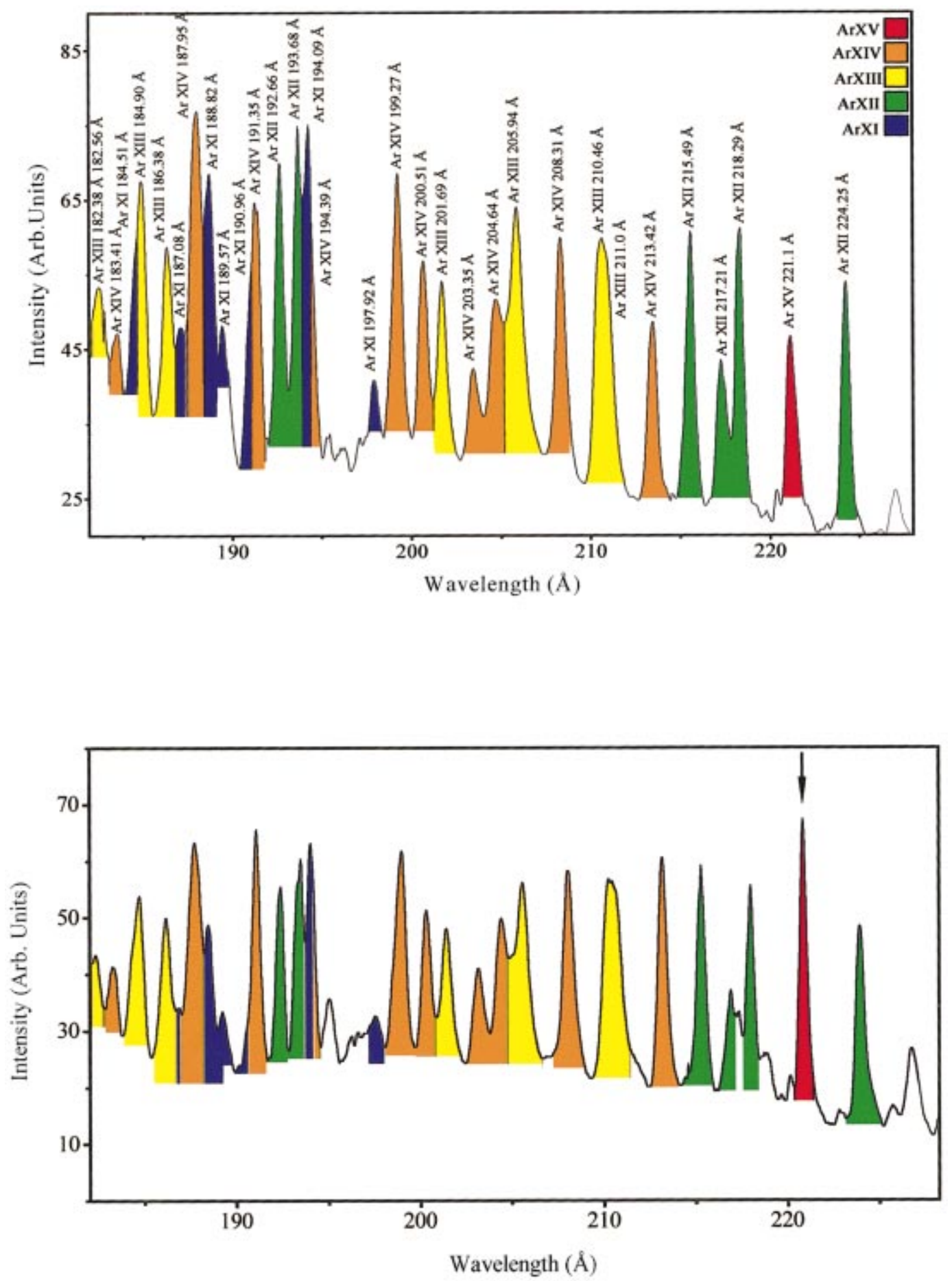

FIG. 5. (Color) Time resolved spectra corresponding to (a) polyacethal capillary, and (b) ceramic capillary obtained at the time of maximum ionization. The peak current was $197 \mathrm{kA}$ with a rise time of $11 \mathrm{~ns}$.

the phenomena of vaporization and ionization to reproduce the phase transformation from solid to vapor and finally to plasma $[16,17]$.

To obtain a consistent picture of the dynamics of the capillary plasma we performed numerous comparisons between the simulations, the experimental spectra, and pinhole images for a large variety of experimental conditions. In the RADEX radiation hydrodynamics the transport of the photons inside the atomic line profiles was modeled in the Biberman-
Holstein approximation [18] which takes advantage of the $1 \mathrm{D}$ symmetry of the capillary plasma. More than 30000 lines were included, of which nearly 3000 are observed in the spectral region between 15 and $23 \mathrm{~nm}$. Two-dimensional edge effects associated with the escape of the plasma from the capillary are not important in such short-pulse highcurrent device, and are negligible for many applications such as soft-x-ray lasers. However, the axial velocity spread at the end of the column somewhat influences the opacity of optical 
ArXV ArXIV ArXIII $\square$ ArXII $\square$ ArXI

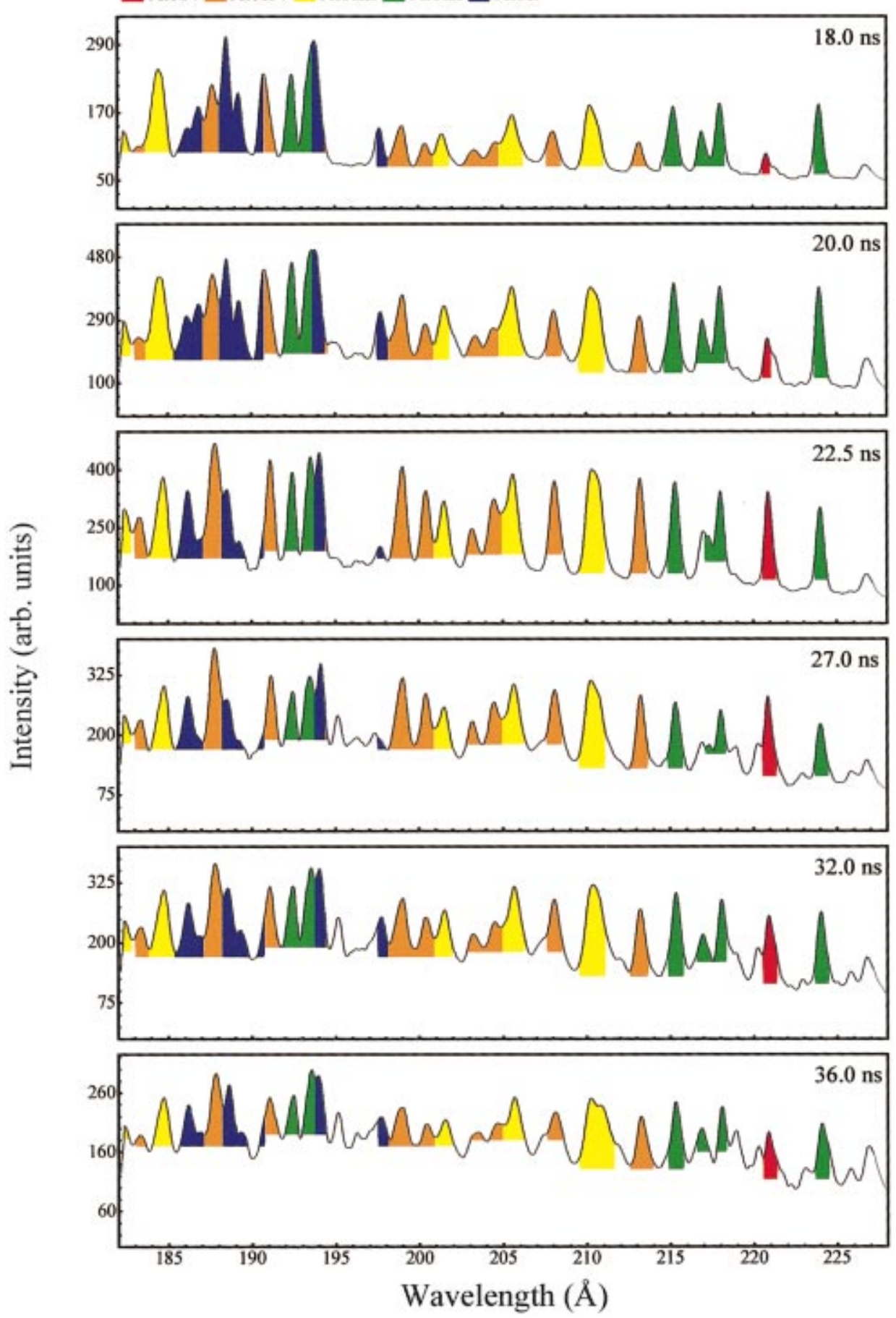

FIG. 6. (Color) Sequence of time resolved spectra corresponding to a $190 \pm 5 \mathrm{kA}$ discharges through a $4 \mathrm{~mm}$ diameter ceramic capillary. The 10-90\% current rise time was $11.5 \pm 1.5 \mathrm{~ns}$. thick lines emitted from the exit region of the capillary facing the spectrometer. Hence, the synthesized spectra corresponding to these conditions cannot be expected to precisely match the measured spectra. Nevertheless, since the main effect of the radiation on the hydrodynamic and atomic kinetics is dominated by the radiation transport in the radial direction, the computed spectra not only show all the lines identified in the experiments, but also reasonably match their intensities.

Note that a similar range of capillary diameters, gas pressures and composition was previously investigated for smaller current discharges $[7,12]$. It was found in the calcu- lations that the major difference between the high current and the lower current cases is the much larger shock-induced plasma temperature change, as it is natural to expect from the much larger amplitude and shorter duration of the current pulse. In this case the plasma speed just before the collapse reaches $2 \times 10^{7} \mathrm{~cm} / \mathrm{s}$ as compared to $6 \times 10^{6} \mathrm{~cm} / \mathrm{s}$ in the case of the $40 \mathrm{kA}$ discharges used in the first Ne-like Ar soft-X-ray laser experiments $[2,7,12]$. The depth of the layer in front of the hot dense shock is of the order of 100-200 $\mu \mathrm{m}$ and is defined by the heat conductivity in the low density gas, and in a lesser extent by the process of electromagnetic field diffusion. The process of heat conduction substantially facili- 


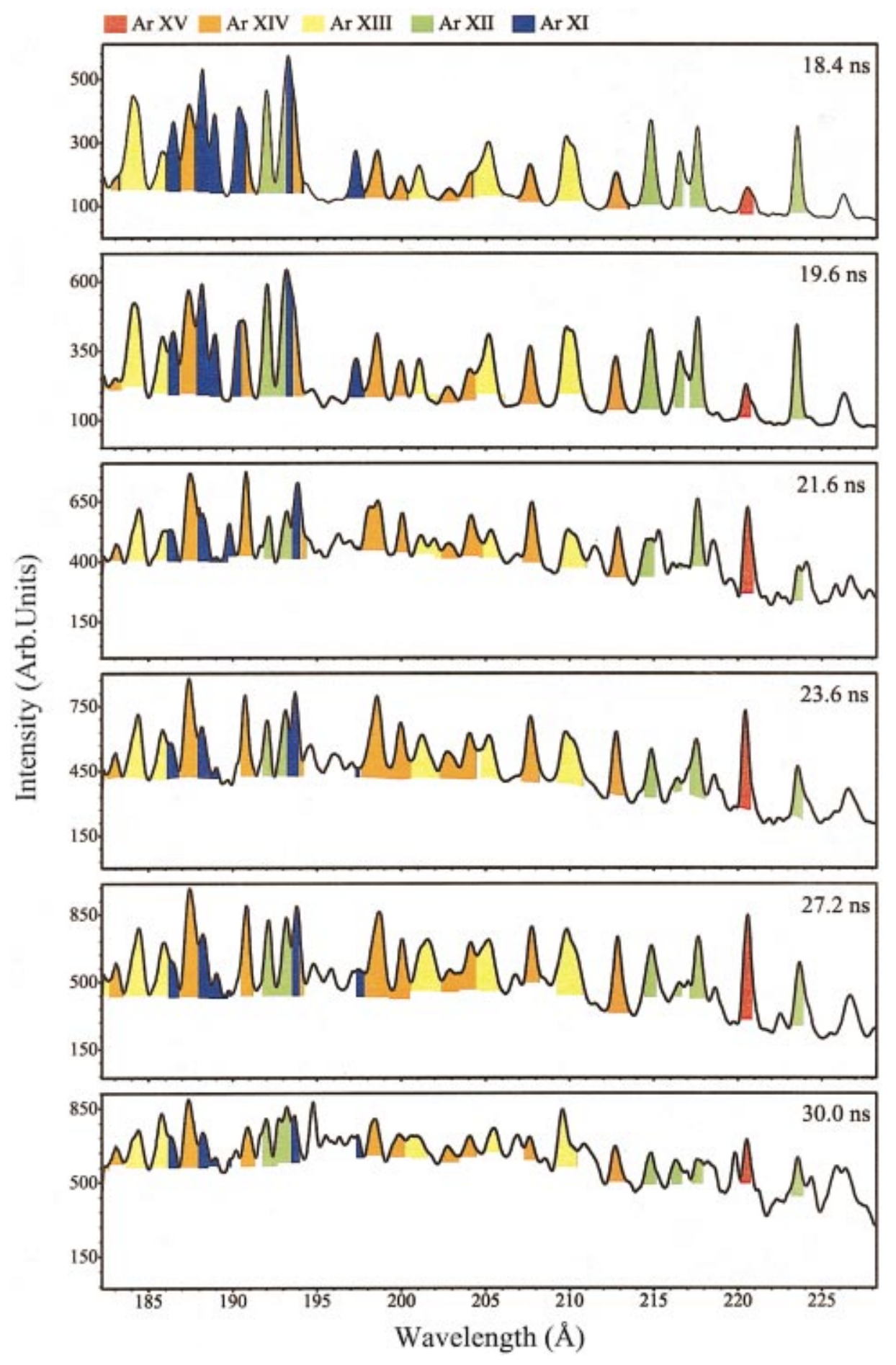

tates the achievement of the high electron temperature resulting in the high degree of ionization observed in the experiment, by introducing additional entropy in the compressed core before the shockwave collapses on axis. Also when the heat wave and the electromagnetic wave arrive on axis, the maximum of the current density and joule dissipation switch to the center of the capillary as in the Ne-like Ar soft-X-ray laser case. Quickly after that, within 1-2 ns, the front of the shock wave arrives to form the hot dense plasma column. Mass continues to be supplied, and after several more ns the plasma starts to cool due to expansion, radiation losses and
FIG. 7. (Color) Sequence of time resolved spectra corresponding to a $190 \pm 5 \mathrm{kA}$ discharges through a $3.3 \mathrm{~mm}$ diameter ceramic capillary. The $10-90 \%$ current rise time was $11.5 \pm 1.5 \mathrm{~ns}$. 


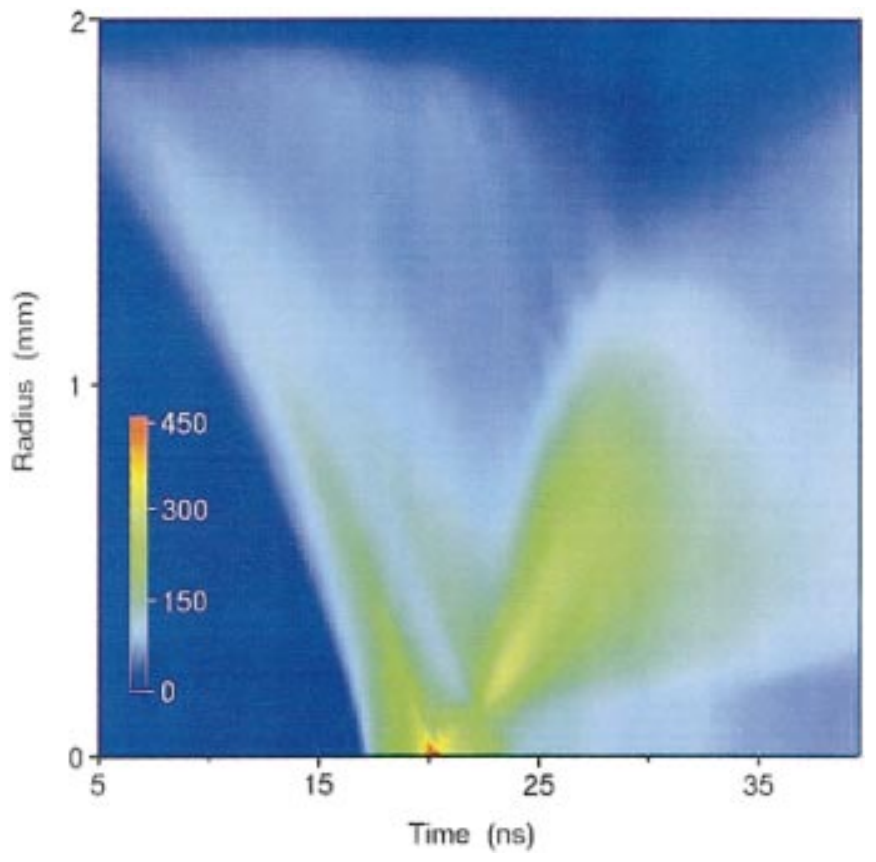

FIG. 8. (Color) Computed spatiotemporal evolution of the electron temperature in a $4 \mathrm{~mm}$ diameter ceramic capillary filled with 1.3 $\mathrm{T}$ of Ar excited by a $195 \mathrm{kA}$ current pulse. The color scale shows electron temperatures in $\mathrm{eV}$.

the entire compression process, substantially facilitate the suppression of the MHD and current instabilities which commonly plague most high current discharge experiments. However, further 2D and 3D numerical modeling is required to determine the main reason of the relatively good stability of these discharges.

The shock wave originates in the vicinity of the capillary surface as the result of the strong ponderomotive $\mathbf{J} \times \mathbf{B}$ force and heating in the skin layer, and then propagates with the speed defined by electromagnetic field diffusion, the Alfven time, and hydrodynamics time. The plasma compression on axis and the dissipative electron viscous heating [20] are computed to induce for a brief period of time a maximum electron temperature as high as 450-500 eV. However, as shown in Fig. 8, this temperature rapidly relaxes to 200-270 $\mathrm{eV}$ in 1-2 ns due to strong ionization and line radiation at electron densities of $(1-2) \times 10^{20} \mathrm{~cm}^{-3}$, which are 3-4 times higher than those obtained in the case of the $40 \mathrm{kA}$ capillary discharges. The electron density reaches its maximum value 3-5 ns after the time of maximum temperature, which corresponds to the minimum observable source size. The nonstationary degree of ionization also reaches its maximum average ion charge of $Z_{s} \sim 15.4$ at this time, and 3-4 ns later the plasma starts to recombine. Figure 9 shows the computed radial profile of the electron temperature and density at the time of maximum compression for the $3.3 \mathrm{~mm}$ diameter ceramic capillary with the same discharge conditions as in Fig. 8. The calculated electron temperature and density on axis are $295 \mathrm{eV}$ and $1.2 \times 10^{20} \mathrm{~cm}^{-3}$, respectively.

The compressed plasma diameter is computed to reach a minimum diameter of $250-300 \mu \mathrm{m}$ at $\sim 19 \mathrm{~ns}$ and $\sim 25 \mathrm{~ns}$ after the initiation of the current pulse in the $3.3 \mathrm{~mm}$ and 4

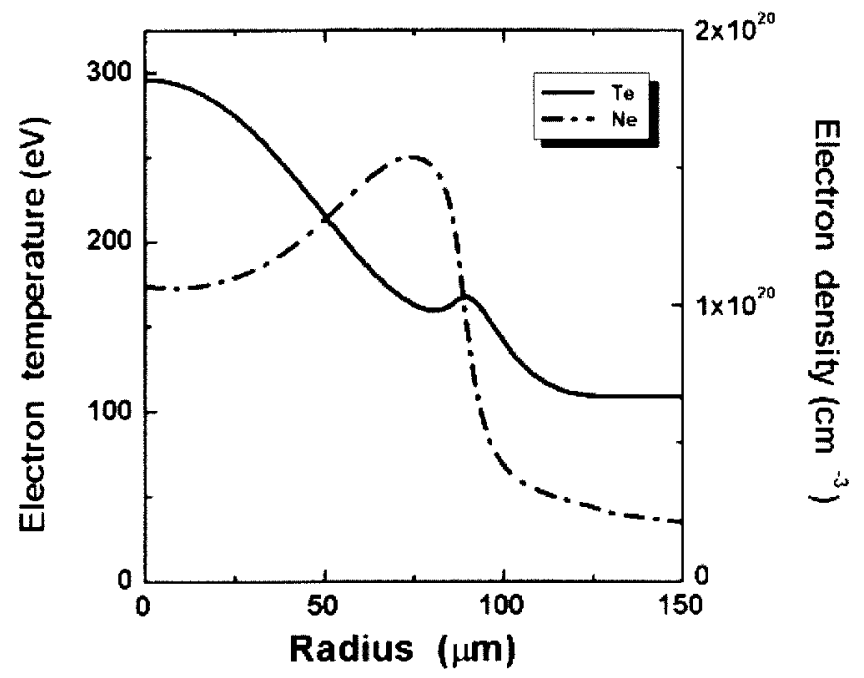

FIG. 9. Computed electron temperature and electron density profiles near the time of maximum compression for a discharge through an argon filled ceramic capillary of $3.3 \mathrm{~mm}$ diameter. The rest of the discharge conditions are equal to those of Fig. 8.

$\mathrm{mm}$ diameter capillaries, respectively. This size of the soft$\mathrm{X}$-ray emitting is in agreement with the observed extent of the soft-x-ray emitting region of the plasma at the time of maximum compression. The simulations also show that a concave electron density profile is formed in the axial region by the reflected shock $\sim 1.5 \mathrm{~ns}$ after the column collapse. This prediction is corroborated by the observation of a depression at the axis of the soft-x-ray emission profiles in the pinhole image acquired a few ns after the maximum compression (see the $28 \mathrm{~ns}$ and $31 \mathrm{~ns}$ frames in Fig. 2). Such kind of electron density profile might be advantageous for some applications, for example waveguiding of soft-x-ray laser beams. Note that, as in previous soft-x-ray laser experiments, in the current experimental setup the time of the plasma column collapse is well matched to the current pulse duration. Hence the energy stored in the driving circuit is well utilized, resulting in efficient plasma generation.

The amount of mass ablated from the capillary wall and involved into the plasma compression process is smaller for the ceramic capillaries than for those made of polyacethal. This is due to several factors, among which are the larger activation energy and thermal conductivity of alumina relative to that of easily thermally decomposed plastic. However, the hydrodynamics of plastic and ceramics wall capillaries were not found to be greatly different. The moment of arrival of the shock wave to the center is similar, 17 and $17.5 \mathrm{~ns}$, respectively, since it is mostly defined by the ponderomotive force and thermal pressure exerted on the plasma in the initial $\sim 10 \mathrm{~ns}$ of the discharge, when the evaporated part of the total mass is very small. In both cases, quickly after the initial stage of the discharge, an additional acceleration of the shock front due to electromagnetic field diffusion takes place in the argon part of the plasma column. This acceleration further minimizes the differences in compression time. The electron temperature is slightly larger in the case of the ceramics capillary, resulting in the more intense ArXV line emission observed in the experiments. This is mainly the 
result of higher plasma heating arising from the nearly two times larger plasma resistivity in the case of the higher $Z$ ceramic capillary plasma [21]. The differences in resistivity do not have a major effect on the overall discharge current, because it is mainly defined by the larger impedance of the generator.

\section{CONCLUSIONS}

In summary, we have generated plasma columns of about $300 \mu \mathrm{m}$ diameter and electron temperatures $>250 \mathrm{eV}$ utilizing fast discharge pulses with a current rise time of $\sim 1.5$ $\times 10^{13} \mathrm{~A} / \mathrm{s}$ and peak currents up to $200 \mathrm{kA}$. Model computations predict plasma column diameter and spectra similar to those observed in the experiments, and suggest the electron densities reach values $>1 \times 10^{20} \mathrm{~cm}^{-3}$. These plasma conditions fulfill the requirements necessary for a variety of possible applications, in particular for attempting the demonstration of discharge-pumped lasers at significantly shorter wavelengths. Of main interest are laser transitions in Ni-like ions, sequence which scales to shorter wavelengths more rapidly than the Ne-like sequence used in the previous capillary discharge soft-x-ray laser demonstrations. For the high electron temperatures and densities that the above results show are achievable with these type of high current capillary discharges, the atomic elements suitable for lasing in the Ni-like sequence range from $Z=42(\mathrm{Mo})$ to $50(\mathrm{Sn})$. The wavelengths of the $4 d{ }^{1} S_{0}-4 p{ }^{1} P_{1}$ transition of these elements cover the region between 18.9 and $11.9 \mathrm{~nm}$. For example, for the $13.2 \mathrm{~nm}$ line of Ni-like CdXXI the gain is calculated to be $\sim 1-2 \mathrm{~cm}^{-1}$, and potentially $>3 \mathrm{~cm}^{-1}$ for optimum discharge parameters. Larger gain coefficients are estimated for Ni-like $\mathrm{Ag}$ XX (13.9 nm) and PdXIX (14.65 $\mathrm{nm})$.

\section{ACKNOWLEDGMENTS}

This work was supported by DARPA Grant No. DE-FG 03-00ER15084 and by the National Science Foundation Grant No. ECS-9713297. Part of the work of V.N.S. and A.L.O. was performed under the auspices of the US Department of Energy at the University of California Lawrence Livermore National Laboratory. The support of the W.M. Keck Foundation to Colorado State University is also gratefully acknowledged.
[1] J. J. Rocca, Rev. Sci. Instrum. 70, 3799 (1999).

[2] J. J. Rocca, V. N. Shlyaptsev, F. G. Tomasel, O. D. Cortazar, D. Hartshorn, and J. L. A. Chilla, Phys. Rev. Lett. 73, 2192 (1994).

[3] B. E. Lemoff, G. Y. Yin, C. L. Gordon III, C. P. J. Barty, and S. E. Harris, Phys. Rev. Lett. 74, 1574 (1995).

[4] D. Korobkin, C. H. Nam, S. Suckewer, and A. Golstov, Phys. Rev. Lett. 77, 5206 (1996); D. Korobkin, et al., ibid. 77, 1476 (1996).

[5] P. V. Nickles, V. N. Shlyaptsev, M. Kalashnikov, M. Schnurer, I. Will, and W. Sadner, Phys. Rev. Lett. 78, 2748 (1997).

[6] F. G. Tomasel, J. J. Rocca, V. N. Shlyaptsev, and C. D. Machietto, Phys. Rev. A 55, 1437 (1996).

[7] J. J. Rocca, D. P. Clark, J. L. A. Chilla, and V. N. Shlyaptsev, Phys. Rev. Lett. 77, 1476 (1996).

[8] J. Dunn, A. L. Osterheld, S. Sheperd, W. E. White, V. N. Shlyaptsev, and R. E. Stewards, Phys. Rev. Lett. 80, 2825 (1998).

[9] M. Frati, M. Seminario, and J. J. Rocca, Opt. Lett. 25, 1022 (2000).

[10] C. D. Machietto, B. R. Benware, and J. J. Rocca, Opt. Lett. 24, 115 (1999).

[11] J. J. Rocca, O. D. Cortazar, B. Szapiro, K. Floyd, and F. G. Tomasel, Phys. Rev. E 47, 1299 (1993).
[12] J. J. Rocca, F. G. Tomasel, M. C. Marconi, V. N. Shlyaptsev, J. L. A. Chilla, B. T. Szapiro, and G. Giudicce, Phys. Plasmas 2, 2547 (1995).

[13] V. N. Shlyaptsev, A. V. Gerusov, A. V. Vinogradov, J. J. Rocca, O. D. Cortazar, F. Tomasel, and B. Szapiro, Proc. SPIE 2012, 99 (1993).

[14] V. N. Shlyaptsev, J. J. Rocca, and A. L. Osterheld, Proc. SPIE 2520, 365 (1995).

[15] M. Marconi, C. Moreno, J. J. Rocca, V. N. Shlyaptsev, and A. Osterheld, Phys. Rev. E 62, 7209 (2000).

[16] Yu. V. Afanasiev and O. N. Krokhin, High Temperature Plasma Phenomena Under the Influence of High Power Radiation with Matter (Mir, Moscow, 1974) (in Russian).

[17] A. A. Samokhin, in Proceedings of the Geberal Physics Institute (Nauka, Moscow, 1998), Vol. 13 (in Russian).

[18] L. M. Biberman, Sov. Phys. JETP 17416 (1947); T. Holstein, Phys. Rev. 72, 1212 (1947); 831159 (1951).

[19] N. A. Bobrova, S. V. Bulanov, T. L. Razinkova, and P. V. Sasorov, Plasma Phys. Rep. 22, 349 (1996).

[20] K. G. Whitney, Phys. Plasmas 6, 816 (1999).

[21] L. Spitzer, Physics of Fully Ionized Gases (Wiley, New York, 1957); S. I. Braginskii, in Transport Processes in Plasmas, edited by M. A. Leontovich (Consultants Bureau, New York, 1963), p. 205. 